Working Paper 05-23

Economics Series 14

April 2005
Departamento de Economía Universidad Carlos III de Madrid

Calle Madrid, 126

28903 Getafe (Spain)

Fax (34) 916249875

\title{
COALITION-PROOF SUPPLY FUNCTION EQUILIBRIA UNDER CAPACITY CONSTRAINTS *
}

\section{Juan Delgado ${ }^{1}$}

\begin{abstract}
Whereas in the absence of capacity constraints the Cournot outcome is the unique coalition-proof supply function equilibrium outcome, the presence of capacity constraints may enlarge the set of equilibrium outcomes. Interestingly, if capacities are sufficiently asymmetric the new equilibrium prices are below the Cournot price. These results have important implications for merger and privatization policies: specifically, capacity divestiture will not necessarily imply lower market prices.
\end{abstract}

Keywords: : Oligopoly, Cournot, competition via supply functions, coalition-proofness, capacity constraints

JEL code: C72, D43, L13, L51

* Acknowledgements. This article is based on the second chapter of my $\mathrm{PhD}$ dissertation. I benefited from the comments and suggestions of Diego Moreno and Bill Hogan. I thank an anonymous referee for helpful comments. Seminar audiences at Carlos III and Harvard are gratefully acknowledged. The author is currently a Repsol YPF fellow at the Harvard John F. Kennedy School of Government. I am grateful to the Repsol YPF-Harvard Fellowship Program for financial support. The opinions expressed in this article are those of the author.

\footnotetext{
${ }^{1}$ John F. Kennedy School of Government - Harvard University, 79 JFK Street, Cambridge, MA 02138, US. Email: Juan_Delgado@harvard.edu
} 


\section{Introduction}

In this paper I study an oligopolistic market where firms compete via supply functions and are subject to capacity constraints. I obtain conclusions substantially different from those that have been obtained when ignoring capacity constraints. Specifically, in the presence of capacity constraints, new equilibria may arise that lead to a lower market price and a larger aggregated output. These results have important implications for the design of regulatory policies, and in particular for antitrust and merger policies.

Consider an industry in which firms compete by simultaneously choosing a (nondecreasing) supply function, and firms are capacity constrained, i.e. they cannot supply beyond a certain quantity. Firms' supply schedules are aggregated to form the market supply function which together with the market demand determines the market clearing price and firms' outputs and profits. This model describes appropriately markets such as, for example, the wholesale spot electricity markets in Spain and in Britain. ${ }^{1}$ In such industries building new capacity takes a long time, and therefore in the meantime firms must make production decision taking capacities as given. In this context, the analysis of games of competition under exogenous capacity constraints is relevant.

Competition via supply functions has been analyzed by several authors -see, e.g., [11], [12], [13], [15], [17]. In the absence of demand uncertainty, the set of market outcomes that can arise in equilibrium is large. ${ }^{2}$ In many traditional industries such as electricity generation there is a small number of firms with a long history in the market. Thus, it is sensible to assume that firms discuss their strategies even if they cannot make binding agreements. Therefore, some of the theoretically feasible equilibrium outcomes might not arise in reality since some (coalitions of) firms may

\footnotetext{
${ }^{1}$ In Britain, the wholesale electriciy market operated in this way until 2001 when the pool was replaced by a new trading system based on bilateral contracts. A similar institution is however still used for balancing supply and residual demand once bilateral contracts have been settled. There are several other countries that to some extent make use of this market institution in restructured electricity markets, e.g., Australia, New Zealand, Nordic countries, Chile and several states of the USA.

${ }^{2}$ Klemperer and Meyer [13] have shown, however, that with unbounded uncertainty equilibrium may be unique.
} 
block them in favor of some other mutually more profitable equilibrium outcomes. In the analysis of such markets, it is therefore important to identify those equilibria which are immune not just to deviations by individual firms but also to profitable deviations by coalitions of firms. In such context, an equilibrium outcome would be therefore more likely to arise if no firm or coalition of firms finds profitable to deviate from such equilibrium. When the firms in the industry take into account the gains they can attain by coordinating their actions, Delgado and Moreno [7] find that the equilibrium multiplicity disappears when the number of firms is above a threshold, and that the Cournot outcome is the unique outcome that can be sustained as an equilibrium.

Green and Newbery [10] study the consequences of introducing capacity constraints in the model with (bounded) demand uncertainty, ${ }^{3}$ and conclude that "the effect of capacity constraints is to narrow the range of feasible [Nash] equilibria and in extreme cases (...) the equilibrium will be unique." (This result also holds when demand is known with certainty.) However, when firms in the industry take into account the gains they can attain by coordinating their actions, the presence of sufficiently asymmetric capacity constraints may increase the number of equilibrium outcomes. Interestingly, these new outcomes involve market prices below the Cournot price. In contrast, when firms' capacities are nearly symmetric the Cournot outcome continues to be the unique outcome that can be sustained as an equilibrium. These results have important implications for antitrust policy and merger control: contrary to common wisdom, more concentrated industries will not necessarily lead to higher prices.

In order to account for the coordination opportunities present in an industry, I use the well-established notion of coalition-proof Nash equilibrium (CPNE) introduced by Bernheim, Peleg and Whinston [3]. This concept has been extensively used for the analysis of coalitional games in a variety of environments -see, e.g., [4], [5], [7], [14], [16]. The concept of coalition-proof Nash equilibrium identifies the strategy profiles for which no coalition of players has an improving and self-enforcing deviation. A deviation is self-enforcing if there is no further self-enforcing and improving deviation available to a proper subcoalition of a deviating coalition. Note that since all indi-

\footnotetext{
${ }^{3}$ Baldick and Hogan [2] provide an analytical solution to a model of competition via supply functions with capacity constraints and analyze the properties and stability of the different possible equilibria.
} 
vidual deviations are self-enforcing, a CPNE is a Nash equilibrium. However, a Nash equilibrium need not be a CPNE.

In the context of this paper, a Nash equilibrium is referred to as a supply function equilibrium (SFE), and a CPNE is referred as a coalition-proof supply function equilibrium (CPSFE). In order to identify the outcomes that can be sustained by CPSFE, I begin by identifying the set of outcomes that can be sustained by SFE in the presence of capacity constraints. I show that any SFE leads to a price not higher than the capacity-constrained Cournot price but strictly higher than the so-called capacity-constrained competitive price. The capacity-constrained Cournot price is the price resulting from firms competing 'a la Cournot' in an industry with capacity constraints. The capacity-constrained competitive price is the minimum feasible price at which no firm produces below its marginal cost.

Next I prove that a coalition-proof Nash equilibria exists in this framework. In particular, I show that the capacity-constrained Cournot outcome can always be sustained by CPSFE, and hence that a CPSFE exists. Moreover, I show that under symmetric capacity constraints, the capacity-constrained Cournot outcome is the unique CPSFE outcome under the conditions established by Delgado and Moreno $[7]{ }^{4}$

When capacities are not symmetric, however, this result does not necessarily hold. Even if the capacities of all firms are above the Cournot output, the conditions that guarantee uniqueness of CPSFE outcomes in the absence of capacity constraints may not guarantee uniqueness when capacity constraints are present. The intuition behind this result is that if at some equilibrium the capacities of a number of firms are binding, the remaining firms can induce higher prices in the residual industry without fearing reactions from the former. Interestingly, the new equilibrium prices are below the Cournot price (which is the highest price that can be sustained by CPSFE.)

Finally, the paper provides necessary and sufficient conditions that guarantee the existence of a unique CPSFE outcome when the market demand and the cost functions are linear. In particular, it is shown that if capacity constraints are sufficiently symmetric, any CPSFE leads to the Cournot outcome. In contrast, if capacities are

\footnotetext{
${ }^{4}$ See Theorem 3.7 in Delgado and Moreno [7].
} 
sufficiently asymmetric other outcomes can be sustained by CPSFE in addition to the Cournot outcome.

These results have interesting policy implications. In particular, they imply that certain asymmetric distributions of capacities may lead to prices below those that arise under symmetric (or nearly symmetric) capacity distributions. The intuition of this result is that in an oligopoly where firms compete via supply functions, larger firms may find more profitable to expand their output (i.e., behaving as Stackelberg leaders on the residual demand) rather than to increase the price.

This is of particular relevance for the design of merger policies and privatization strategies. In the case of merger analysis, the use of concentration indexes might be misleading since they may not be directly related to the market price (even in the absence of efficiency gains.) Mergers leading to more symmetric market structures may result in higher prices than mergers resulting in more asymmetric market structures. Similarly, a policymaker facing the break-up of a monopoly may wish to favor asymmetric distributions of capacities rather than symmetric ones in order to induce lower market prices. However, not all asymmetric distributions of capacities will achieve this goal. This will be further developed in the last section of the paper.

\section{Supply Function Equilibria}

\subsection{The industry}

Consider an oligopolistic industry where firms compete in the production of a homogeneous good. The industry demand, $D: \mathbb{R}_{+} \rightarrow \mathbb{R}_{+}$, is known to all firms with certainty. ${ }^{5}$ Throughout it is assumed that $D$ is a twice continuously differentiable strictly decreasing and concave function ${ }^{6}$ on $(0, \rho)$, where $\rho>0$ satisfies $D(p)>0$ for $p<\rho$, and $D(p)=0$ for $p \geq \rho$. All firms have access to the same technology, and therefore have identical cost function, $C: \mathbb{R} \rightarrow \mathbb{R}$; I assume that $C$ is twice continuously differentiable, non-decreasing and convex on $\mathbb{R}_{+}$, and satisfies $C^{\prime}(0)<\rho$,

\footnotetext{
${ }^{5}$ In many markets the demand can be anticipated with a great accuracy, and therefore uncertainty plays a small role. This is the case, for example, in the spot electricity market in Spain where each firm submits a bid for each of the ( 24 one-hour) periods in which the market is divided.

${ }^{6}$ Concavity of demand is not strictly required for the profit function to be concave but is assumed for technical convenience.
} 
and $C(q)=C(0)$ for $q \leq 0$. (Extending the domain of the cost function to include negative quantities is inconsequential and simplifies our analysis.) Write $N$ for the set of firms $\{1, \ldots, n\}$, where $n \geq 2$. Firms are capacity-constrained in the sense that Firm $i$ cannot produce above a certain quantity $k_{i}>0$.

An industry is therefore described by the vector $(D, C, N, k)$, indicating the market demand, $D$, the firms' cost function, $C$, the set of firms $N$ and the firm's capacities $k=\left(k_{1}, k_{2}, \ldots, k_{n}\right)$. Without loss of generality, let $k_{i} \geq k_{i+1} \forall i \in\{1, \ldots, n-1\}$. In what follows, let us be given an industry $(D, C, N, k)$.

\subsection{Equilibria}

Firms compete by simultaneously choosing a function $r_{i}(p)$; where $r_{i}(p)$ is a nondecreasing real-valued function on $[0, \rho]$ continuously differentiable on $(0, \rho)$. Firm $i$ 's supply function $s_{i}(p)$ is equal to $r_{i}(p)$ for $r_{i}(p)<k_{i}$ and to $k_{i}$ otherwise, i.e.,

$$
s_{i}(p)=\left\{\begin{array}{c}
r_{i}(p) \text { if } r_{i}(p)<k_{i} \\
k_{i} \text { otherwise }
\end{array}\right.
$$

For a profile of supply functions $s=\left(s_{1}, \ldots, s_{n}\right)$, a market clearing price is a solution to the equation

$$
\sum_{i=1}^{n} s_{i}(p)=D(p) .
$$

The assumptions on the market demand and the firms' supply functions guarantee that if a market clearing price exists, then it is unique ${ }^{7}$. For each profile of supply functions $s$, let $p(s)$ be the market clearing price if it exists, and let $p(s)$ be zero if a market clearing price does not exist. Firm $i$ 's profits (payoff) are given by $\pi_{i}(s)=$ $p(s) s_{i}(p(s))-C\left(s_{i}(p(s))\right)$. (This construction implicitly assumes that firms' revenues are zero when a market clearing price does not exist.)

A supply function equilibrium (SFE henceforth) is a (pure strategy) Nash equilibrium of the game described. Write $\operatorname{SFE}(D, C, N, k)$ for the set of supply function equilibria. In a SFE each firm maximizes profits, subject to its capacity constraint, on its "residual demand". Let $\hat{s} \in S F E(D, C, N, k)$ and $p(\hat{s})=\hat{p}$. Write

\footnotetext{
${ }^{7}$ In contrast to traditional capacity-constrained oligopoly models in which consumers are allowed to buy at different prices and, thus, a rationing rule has to be specified, here the market price is determined by the market clearing price and all transactions are made at the same price.
} 
$N(\hat{s})=\left\{i \in N \mid \hat{s}_{i}(\hat{p})<k_{i}\right\}$ and $n(\hat{s})=\# N(\hat{s})$. Then, for each $i \in N, \hat{p}$ solves

$$
\begin{aligned}
& \max _{p \in[0, \rho]} p\left(D(p)-\sum_{j \neq i} \hat{s}_{j}(p)\right)-C\left(D(p)-\sum_{j \neq i} \hat{s}_{j}(p)\right) \\
& \text { s.t. } \quad D(p)-\sum_{j \neq i} \hat{s}_{j}(p) \leq k_{i} .
\end{aligned}
$$

If $\hat{p} \in(0, \rho)$, then, for each $i \in N, \hat{s}$ satisfies

$$
\begin{aligned}
D(\hat{p})-\sum_{j \neq i} \hat{s}_{j}(\hat{p})+ & \\
& +\left(D^{\prime}(\hat{p})-\sum_{\substack{j \neq i \\
j \in N(\hat{s})}} \hat{r}_{j}^{\prime}(\hat{p})\right)\left(\hat{p}-C^{\prime}\left(D(\hat{p})-\sum_{j \neq i} \hat{s}_{j}(\hat{p})\right)-\lambda_{i}\right)=0,
\end{aligned}
$$

where $\lambda_{i} \geq 0\left(\lambda_{i}=0\right.$ if $\left.D(\hat{p})-\sum_{j \neq i} \hat{s}_{j}(\hat{p})<k_{i}\right)$. Using the market clearing condition $(M C)$ and writing $\hat{q}_{i}=D(\hat{p})-\sum_{j \neq i} \hat{s}_{j}(\hat{p})$, this condition can be written, for each $i \in N$, as

$$
\hat{q}_{i}+\left(D^{\prime}(\hat{p})-\sum_{\substack{j \neq i \\ j \in N(\hat{s})}} \hat{r}_{j}^{\prime}(\hat{p})\right)\left(\hat{p}-C^{\prime}\left(\hat{q}_{i}\right)-\lambda_{i}\right)=0,
$$

where $\lambda_{i} \geq 0$. If in addition each $\hat{r}_{i}$ is a convex function, then satisfying $E_{i}$ for $i \in N$ is a sufficient condition for a strategy profile $\hat{s}$ to be a SFE.

If Firm $i$ 's capacity is binding at the equilibrium price $\hat{p}$, condition $E_{i}$ becomes

$$
k_{i}+\left(D^{\prime}(\hat{p})-\sum_{\substack{j \neq i \\ j \in N(\hat{s})}} \hat{r}_{j}^{\prime}(\hat{p})\right)\left(\hat{p}-C^{\prime}\left(k_{i}\right)-\lambda_{i}\right)=0,
$$

where $\lambda_{i} \geq 0$. If Firm $i$ 's capacity is not binding at the equilibrium price $\hat{p}$, condition $E_{i}$ becomes

$$
\hat{q}_{i}+\left(D^{\prime}(\hat{p})-\sum_{\substack{j \neq i \\ j \in N(\hat{s})}} \hat{r}_{j}^{\prime}(\hat{p})\right)\left(\hat{p}-C^{\prime}\left(\hat{q}_{i}\right)\right)=0 .
$$

For $s \in S F E(D, C, N, k)$, denote by $\left(p(s), q_{1}(s), \ldots, q_{n}(s)\right)$ the associated market outcome.

Next, the definitions of the Cournot price and the competitive price are adapted to the scenario with capacity constraints. Those prices will play an important role in determining the set of prices that can be sustained by SFE. 
In the industry without capacity constraints $(D, C, N)$, the Cournot outcome $\left(\bar{p}, \bar{q}_{1}, \ldots, \bar{q}_{n}\right)$ is characterized by the system of equations ${ }^{8}$

$$
\bar{q}_{i}+\left(\bar{p}-C^{\prime}\left(\bar{q}_{i}\right)\right) D^{\prime}(\bar{p})=0, \quad \forall i \in N
$$

and

$$
\sum_{i=1}^{n} \bar{q}_{i}=D(\bar{p})
$$

Given the industry $(D, C, N, k)$, define $N_{i}=\{1, \ldots, i\}$ and let $D_{k, N_{i}}(p)$ be the residual demand when the set of firms $N \backslash N_{i}=\{i+1, \ldots, n\}$ produce at full capacity. Thus $D_{k, N_{i}}$ is given for $p \in \mathbb{R}_{+}$by

$$
D_{k, N_{i}}(p)= \begin{cases}D(p)-\sum_{j>i} k_{j} & \text { if } D(p)-\sum_{j>i} k_{j} \geq 0 \\ 0 & \text { otherwise. }\end{cases}
$$

Also define

$$
\bar{N}=\left\{j \in N \mid k_{j}>\bar{q}\left(D_{k, N_{j}}, C, N_{j}\right)\right\} .
$$

Note that since by definition $k_{j} \geq k_{i}$ for $j<i$, then $i \in \bar{N}$ implies $j \in \bar{N}$ for $j<i$. Let $\bar{n}=\max \{j \in \bar{N}\}$ if $\bar{N} \neq\{\emptyset\}$, and $\bar{n}=0$ if $\bar{N}=\{\emptyset\}$. Thus, $\bar{N}=\{i \in N \mid i \leq \bar{n}\}$ is the set of firms which capacities are strictly larger than the Cournot output in the (residual) industry $\left(D_{k, \bar{N}}, C, \bar{N}\right)$. Likewise, $N \backslash \bar{N}=\{i \in N \mid i>\bar{n}\}$ is the set of firms that do not have sufficient capacity to produce the Cournot quantity in the industry $\left(D_{k, \bar{N}}, C, \bar{N}\right)$ for $i \geq \bar{n}+1$. If $\bar{n}=0$ then no firm has enough capacity to produce the Cournot quantity in the industry $\left(D_{k, N_{j}}, C, N_{j}\right)$ for every possible value of $j$. In particular, this will happen when the largest firm does not have enough capacity to produce the profit maximizing quantity in the residual industry where all firms but the largest firm produce all their capacity.

Thus, $\forall i \leq \bar{n}$, Firm $i$ has enough capacity to produce more than the Cournot quantity when firms in $N \backslash \bar{N}=\{\bar{n}+1, \ldots, n\}$ produce at full capacity.

In the industry $(D, C, N, k)$, a capacity-constrained Cournot outcome $\left(\bar{p}, \bar{q}_{1}, \ldots, \bar{q}_{n}\right)$ is characterized by the system of equations

$$
\bar{q}_{i}+\left(\bar{p}-C^{\prime}\left(\bar{q}_{i}\right)\right) D^{\prime}(\bar{p})=0, \quad \text { for } \quad i \in \bar{N},
$$

\footnotetext{
${ }^{8}$ These equations characterize the Cournot equilibrium in a quantity-setting oligopoly game.
} 


$$
\bar{q}_{i}=k_{i}, \quad \text { for } \quad i \in N \backslash \bar{N}
$$

and

$$
\sum_{i=1}^{n} \bar{q}_{i}=D(\bar{p}) .
$$

Equations $\bar{C} 1 k$ and $\bar{C} 2 k$ ensure that each firm maximizes profits, whereas $\bar{C} 3 k$ ensures that the market clears. The assumptions on demand and cost functions imply the existence of a unique capacity-constrained Cournot outcome, which is symmetric for all $i \leq \bar{n}$ (i.e., satisfies $\bar{q}_{1}=\ldots=\bar{q}_{\bar{n}}=\bar{q}$ ).

Given an industry $(D, C, N, k)$, I denote by $\bar{p}(D, C, N, k)$ the capacity-constrained Cournot price. The capacity-constrained Cournot price coincides with the equilibrium price resulting from firms competing 'a la Cournot' in a capacity-constrained industry. Therefore, if $N \equiv \bar{N}$, then $\bar{p}(D, C, N, k)=\bar{p}(D, C, N)$ and $\bar{q}_{i}(D, C, N, k)=$ $\bar{q}(D, C, N)$, whereas if $\bar{N}=\{\emptyset\}$ then $\bar{p}(D, C, N, k)$ is the solution to the equation $\sum_{i=1}^{n} k_{i}=D(\bar{p})$. Note also that $\bar{p}(D, C, N, k)=\bar{p}\left(D_{k, \bar{N}}, C, \bar{N}\right)$, i.e. the capacityconstrained Cournot price for the industry $(D, C, N, k)$ coincides with the Cournot price of the industry $\left(D_{k, \bar{N}}, C, \bar{N}\right)$.

For $i \in \bar{N}$, let $\bar{q}_{i}(D, C, N, k)=\bar{q}(D, C, N, k) .{ }^{9}$ Note that $\bar{q}(D, C, N, k)=\bar{q}\left(D_{k, \bar{N}}, C, \bar{N}\right)$.

In the industry without capacity constraints $(D, C, N)$, a competitive outcome $\left(\underline{p}, \underline{q}_{1}, \ldots, \underline{q}_{n}\right)$ satisfies the system of equations

$$
C^{\prime}\left(\underline{q}_{i}\right)=\underline{p}
$$

for $i \in N$, and

$$
\sum_{i=1}^{n} \underline{q}_{i}=D(\underline{p}) .
$$

Given the industry $(D, C, N, k)$, define

$$
\underline{N}=\left\{j \in N \mid k_{j}>\frac{1}{j} D_{k, N_{j}}\left(\underline{p}\left(D_{k, N_{j}}, C, N_{j}\right)\right)\right\} .
$$

Again, note that since $k_{j} \geq k_{i}$ for $j<i, i \in \underline{N}$ implies $j \in \underline{N}$ for $j<i$. Let $\underline{n}=\max \{j \in \underline{N}\}$ if $\underline{N} \neq\{\emptyset\}$ and $\underline{n}=0$ if $\underline{N}=\{\emptyset\}$. If $\underline{n}>0$, the capacity-constrained competitive price $\underline{p}(D, C, N, k)$ is defined by $\underline{p}(D, C, N, k)=\underline{p}\left(D_{\underline{N}}, C, \underline{N}\right)$. If $\underline{n}=0$,

\footnotetext{
${ }^{9}$ Note that for $i \in \bar{N}$ the Cournot outcome is symmetric.
} 
the capacity-constrained competitive price $\underline{p}(D, C, N, k)$ is defined by $\underline{p}(D, C, N, k)=$ $D^{-1}\left(\sum_{i=1}^{n} k_{i}\right)$.

Clearly,

$$
0 \leq \underline{p}(D, C, N, k) \leq \bar{p}(D, C, N, k)<\rho .
$$

\section{$2.3 \quad$ Results}

Write $S F E_{p}(D, C, N, k)$ for the set of prices that can be sustained by $\mathrm{SFE}$, i.e. if $p \in S F E_{p}(D, C, N, k)$ then there exists $s \in S F E(D, C, N, k)$ such that $p(s)=p$.

Theorem 2.1 below establishes that the set of prices that can be sustained by SFE in an industry with capacity constraints is the half open interval containing the prices between the capacity-constrained competitive price and capacity-constrained Cournot price. The proof can be easily adapted from the proof of Theorem 2.2 in [7] and is omitted.

Theorem 2.1. $S F E_{p}(D, C, N, k)=(\underline{p}(D, C, N, k), \bar{p}(D, C, N, k)]$.

Delgado and Moreno [7] show that in the absence of capacity constraints the set of prices that can be sustained is $(\underline{p}(D, C, N), \bar{p}(D, C, N)]$. Since $\underline{p}(D, C, N) \leq$ $\underline{p}(D, C, N, k)$ and $\bar{p}(D, C, N) \leq \bar{p}(D, C, N, k)$, both the lower and the upper bound of the equilibrium price set may increase in the presence of capacity constraints. Therefore, in the presence of capacity constraints higher prices might be sustained by SFE. As stated by Corollary 2.2, in the extreme case where $\bar{N} \neq\{\emptyset\}, \underline{p}(D, C, N, k)=$ $\bar{p}(D, C, N, k)$, i.e. the equilibrium outcome will be unique.

Corollary 2.2. Let $(D, C, N, k)$ be an industry. If $\bar{N}=\{\emptyset\}$, then every $s \in$ $\operatorname{SFE}(D, C, N, k)$ satisfies $p(s)=D^{-1}\left(\sum_{i=1}^{n} k_{i}\right)$ and $q_{i}(s)=k_{i}$ for $i \in N$.

The proof derives trivially from Theorem 2.1. When $\bar{N} \neq\{\emptyset\}$ no firm will have sufficient capacity to produce the unconstrained Cournot quantity and therefore, at any equilibrium outcome every firm will produce all its capacity, i.e. $q_{i}(s)=k_{i}$. Thus, the set of prices that can be sustained by SFE will be reduced to $\bar{p}(D, C, N, k)=$ $D^{-1}\left(\sum_{i=1}^{n} k_{i}\right)$. 


\section{Coalition-Proof Supply Function Equilibria}

In this section the concept of coalition-proof supply function equilibrium is introduced and it is shown that under capacity constraints a coalition-proof supply function equilibrium exists.

\subsection{The notion of coalition-proof supply function equilibrium}

The notion of coalition-proof supply function equilibrium (CPSFE) identifies the SFE which are self-enforcing in the stronger sense that neither individual firms nor coalitions of firms have credible improving deviations. The notion of CPSFE is an adaptation of the concept of coalition-proof Nash equilibrium introduced by Bernheim, Peleg and Whinston [3]. ${ }^{10}$ The concept of coalition-proof Nash equilibrium relates to Aumann's concept of strong Nash equilibrium (see [1]). A strategy profile is a strong equilibrium if no coalition can profitably deviate from the prescribed profile. The concept of coalition-proof Nash equilibrium limits the set of admissible deviations: only those deviations which are immune to further deviations of subcoalitions are valid deviations. ${ }^{11}$

The following formal definition adapts the concept of CPSFE defined in [7] to industries with capacity constraints.

Let $(D, C, N, k)$ be an industry. Denote by $2^{N}$ the set of all possible coalitions. For a strategy profile $s$ and a coalition $M \in 2^{N}$, write $s_{M}$ for the profile of supply functions of the members of $M$, and write $m$ for the cardinality of the set $M$. Let $s$ be a strategy profile and let $M \in 2^{N}, 2 \leq m<n$, be a coalition of firms (recall that

\footnotetext{
${ }^{10}$ See also [4] for the application of this concept to the Cournot quantity competition game and to several other well-known games. Delgado and Moreno [7] use this concept in a market where firms complete via supply functions. Chowdhury and Sengupta [5] analyse the existence of CPNE in a Bertrand price competition environment.

${ }^{11}$ The notion of coalition-proof Nash equilibrium is the most widely used noncooperative coalitional equilibrium concept. The concept is not, however, absent of difficulties and criticisms. The existence of a Coalition-proof Nash Equilibrium, for example, is difficult to prove even in simple games (see [4].) One of the main criticisms to this concept is that it assumes passive behavior on the part of coalitions complementary to the deviating coalition. Moreover there is not a dynamic concept of CPNE which is generally accepted (see e.g. [9] for a discussion and a possible definition of CPNE in extensive form games.)
} 
$n \geq 2$ ). Holding fixed the strategies of the members of the complementary coalition, $s_{N \backslash M}$, the situation the group of firms in $M$ faces can be modeled as that of an "industry" $\left(D_{s, M}, C, M, k\right)$, where $D_{s, M}$ is given for $p \in \mathbb{R}_{+}$by

$$
D_{s, M}(p)= \begin{cases}D(p)-\sum_{i \in N \backslash M} s_{i}(p) & \text { if } D(p)-\sum_{i \in N \backslash M} s_{i}(p) \geq 0, \\ 0 & \text { otherwise. }\end{cases}
$$

This recursive structure allows us to formalize the notion of CPSFE.

Coalition-Proof Supply Function Equilibrium: Let $(D, C, N, k)$ be an industry.

(1) If $n=2$, a coalition-proof supply function equilibrium is a strategy profile $s \in$ $\operatorname{SFE}(D, C, N, k)$ such that there is no $\tilde{s} \in \operatorname{SFE}(D, C, N, k)$ satisfying $\pi_{i}(\tilde{s})>\pi_{i}(s)$ for $i \in N$.

(2) Let $n>2$ and assume that the notion of coalition-proof supply function equilibrium has been defined for industries with fewer than $n$ firms.

(i) A strategy profile $s$ is self-enforcing if $s \in S F E(D, C, N, k)$, and if for all $M \in 2^{N}, 2 \leq m<n, s_{M}$ is a coalition-proof supply function equilibrium of the industry $\left(D_{s, M}, C, M, k\right)$.

(ii) A strategy profile $s$ is a coalition-proof supply function equilibrium if it is self-enforcing and if there is no self-enforcing strategy $\tilde{s}$ such that $\pi_{i}(\tilde{s})>\pi_{i}(s)$ for $i \in N$.

The definition of CPSFE applies to industries with no fewer than two firms. Note that a CPSFE is a SFE, and therefore it is invulnerable to deviations by a single firm. Given an industry $(D, C, N, k)$, write $\operatorname{CPSFE}(D, C, N, k)$ for the set of coalitionproof supply function equilibria.

\subsection{Existence}

Theorem 3.1 establishes that every capacity-constrained industry has a CPSFE. In fact, it is shown that the capacity-constrained Cournot outcome can be sustained by CPSFE. Theorem 3.1 below is an extension of Theorem 3.1 in [7] for industries with capacity constraints.

Theorem 3.1. Every industry $(D, C, N, k)$ has a coalition-proof supply function equilibrium. Moreover, there is $s \in C P S F E(D, C, N, k)$ such that $p(s)=\bar{p}(D, C, N, k)$. 


\section{Uniqueness of coalition-proof supply function equi- librium outcome}

Delgado and Moreno [7] study conditions under which the Cournot outcome is the unique outcome that can be sustained by a CPSFE in the industry without capacity constraints $(D, C, N)$. In particular, they show that under appropriate conditions on the demand and cost functions, the number

$$
n^{*}=2+\frac{D^{\prime}(\bar{p})+D^{\prime \prime}(\bar{p})\left(\bar{p}-C^{\prime}(\bar{q})\right)}{D^{\prime}(\bar{p})\left(1-D^{\prime}(\bar{p}) C^{\prime \prime}(\bar{q})\right)}
$$

where $\bar{p}=\bar{p}(D, C, N)$, provides a threshold on the number of firms that guarantees that if $n \geq n^{*}$, then the Cournot outcome is the unique outcome that can be sustained by CPSFE in the industry $(D, C, N)$. For example, if the demand function is linear, $n^{*}=3$.

Henceforth, it is assumed that $D$ and $C$ satisfy the appropriate conditions that guarantee that for $n \geq n^{*}$ the Cournot outcome is the unique outcome that can be sustained by CPSFE in the industry without capacity constraints $(D, C, N) .{ }^{12}$

In the next subsections I will show that this condition is not sufficient to guarantee the uniqueness result in the presence of capacity constraints. It will be shown that if capacities are symmetric then $n \geq n^{*}$ guarantees the uniqueness of CPSFE outcome under capacity constraints. However, as it will be also be shown, in the presence of asymmetric capacity constraints this condition does not always guarantee the above result.

\subsection{Symmetric capacities}

In this subsection I show that under symmetric capacity constraints there is a unique CPSFE outcome. Therefore the presence of symmetric capacity constraints does not alter the result of existence of a unique CPSFE outcome which coincides with the Cournot outcome.

Proposition 4.1 below shows that if firms' capacities are symmetric, then the unique outcome that can be sustained by CPSFE is the capacity-constrained Cournot

\footnotetext{
${ }^{12}$ Such conditions relate to the curvature of the demand and cost functions. See Theorem 3.7 in Delgado and Moreno [7].
} 
outcome. The proof of this proposition is given in the Appendix.

Proposition 4.1. Let $(D, C, N, k)$ be an industry. If capacities are symmetric and $n \geq n^{*}$ then

$$
\left(p(s), q_{1}(s), \ldots, q_{n}(s)\right)=(\bar{p}(D, C, N, k), \bar{q}(D, C, N, k), \ldots, \bar{q}(D, C, N, k))
$$

for all $s \in C P S F E(D, C, N, k)$.

If capacities are below the Cournot output there is a unique SFE outcome and therefore Proposition 4.1 derives trivially from Corollary 2.2 and Theorem 3.1. If capacities are above the 'unconstrained' Cournot output, the above proposition indicates that capacity constraints play no role at the unique equilibrium outcome and the equilibrium outcome of the game with capacity constraints thus coincides with that of the game without capacity constraints.

As in the absence of capacity constraints, under symmetric capacity constraints $n \geq n^{*}$ is a sufficient condition for the existence of a unique CPSFE outcome. This means that the Cournot quantity-setting model provides a "reduced form" of a "structural model" where capacity-constrained symmetric firms compete via supply functions taking into account the coordination possibilities present in the industry.

\subsection{Asymmetric capacities}

This subsection analyses whether the existence of a unique CPSFE outcome holds under asymmetric capacity constraints. As it will be shown, this will not always be the case even if every firm in the industry has enough capacity to produce the Cournot quantity. Surprisingly, under some asymmetric distributions of capacities, CPSFE outcomes other than Cournot may yield. Since the Cournot price is the highest equilibrium price, such new CPSFE outcomes will necessarily imply prices lower than the Cournot price. In this subsection I identify the necessary and sufficient conditions that guarantee the existence of a unique CPSFE outcome in an industry with linear demand and cost functions. A result that plays an important role in the search of these conditions is the fact that in any CPSFE resulting in an outcome other than Cournot there must be at least $n-n^{*}$ firms with binding capacities at 
such equilibrium (which implies that if the capacity of the smallest $n-n^{*}$ firms is sufficiently large then no CPSFE resulting in an outcome other than Cournot exists.)

It is easy to construct examples where $n \geq n^{*}>\bar{n}$ (i.e., the number of firms with above-Cournot capacity is less than $n^{*}$ ) and there are outcomes other than the capacity-constrained Cournot outcome that can be sustained by CPSFE. Consider the industry $(D, C, N, k)$ where $\bar{n}<n^{*}$ and let $s \in S F E(D, C, N, k)$ be such that $q_{i}(s)=k_{i} \forall i>\bar{n}$. Since uniqueness of CPSFE outcomes cannot be guaranteed in the "residual industry" $\left(D_{k, \bar{N}}, C, \bar{N}, k\right)$. for $\bar{n}<n^{*}$, then uniqueness of CPSFE outcomes cannot be guaranteed in $(D, C, N, k)$ either, even though $n \geq n^{*}$.

Further, neither the condition $n \geq \bar{n} \geq n^{*}$ guarantees the uniqueness of CPSFE outcomes, as Example 4.2 below shows. Note that in this example all firms in the industry have enough capacity to produce the Cournot quantity. Thus, the uniqueness result established by Delgado and Moreno [7] does not necessarily hold under asymmetric capacities.

Example 4.2. Consider the industry $(D, C,\{1,2,3\}, k)$ where $C(q)=0$ for $q \in \mathbb{R}$, $D(p)=100-p$ for $p \in[0,100]$, and $k=(35,30,28)$. Note that $\bar{p}(D, C, N, k)=$ $\bar{p}(D, C, N)=25$ and $\bar{q}(D, C, N, k)=\bar{q}(D, C, N)=25$.

Consider the strategy $\hat{s}$ such that

$$
\begin{aligned}
& \hat{s}_{1}(p)=30 \forall p, \\
& \hat{s}_{2}(p)=\left\{\begin{array}{c}
12+\frac{3}{7} p \text { if } p<42, \\
30 \text { if } p \geq 42,
\end{array}\right. \\
& \hat{s}_{3}(p)=28 \quad \forall p .
\end{aligned}
$$

It is easy to prove that $\hat{s} \in S F E(D, C,\{1,2,3\}, k)$ where

$$
\left(p(\hat{s}), q_{1}(\hat{s}), q_{2}(\hat{s}), q_{3}(\hat{s})\right)=(21,30,21,28)
$$

Firms' profits are $\pi_{1}(\hat{s})=630>\bar{\pi}=625, \pi_{2}(\hat{s})=441$ and $\pi_{3}(\hat{s})=588$.

In the Appendix it is shown that $\hat{s} \in \operatorname{CPSFE}(D, C,\{1,2,3\}, k)$. For this, it is shown that $\hat{s}$ is self-enforcing and that there is no self-enforcing strategy $\tilde{s}$ such that $\pi_{i}(\tilde{s})>\pi_{i}(\hat{s})$ for $i \in N$.

Note that since the Cournot price is the highest price that can be sustained by SFE, any alternative CPSFE will necessarily imply a price lower than the Cournot 
price. Thus, some forms of asymmetry might be desirable from a welfare perspective since they may allow for the possibility of existence of lower equilibrium prices. ${ }^{13}$ This will be further commented in the final section.

Having shown that the presence of asymmetric capacity constraints may invalidate the uniqueness of CPSFE outcomes I proceed to investigate which additional conditions are needed in order to guarantee the existence of a unique CPSFE outcome.

Proposition 4.3 below specifies how any additional CPSFE outcome will look like. In particular, the proposition shows that any SFE at which $n^{*}$ or more firms produce under capacity cannot be CPSFE. The proof of this proposition is given in the Appendix.

Proposition 4.3. Let $(D, C, N, k)$ be an industry such that $n \geq n^{*}$. If $s \in C P S F E(D, C, N, k)$ such that $p(s)<\bar{p}(D, C, N, k)$, then $n-n(s)<n^{*}$.

The idea behind the proof of the above proposition is the following: assume there is an SFE at which the capacities of the coalition $N^{\prime} \subseteq N$ (where $\# N^{\prime} \geq n^{*}$ ) are not binding. Thus, the deviation of the coalition formed by the firms in $N^{\prime}$ to a SFE resulting in the Cournot outcome is self-enforcing and implies larger profits for all the members of the coalition. Thus, the initial SFE cannot be a CPSFE.

The previous result restricts the search of additional CPSFE to the family of SFE outcomes in which less than $n^{*}$ firms produce under their capacity.

Next, I analyze the conditions under which CPSFE outcome uniqueness holds in a capacity-constrained linear industry. A capacity-constrained linear industry is described by a linear demand function, (i.e., $D(p)=a-b p$, for $p \in\left[0, \frac{a}{b}\right]$, where $a, b \in \mathbb{R}_{+}$), and a linear cost function (i.e., $C(q)=c q$, for $q \geq 0$, where $c \in \mathbb{R}_{+}$). The assumption that $C^{\prime}(0)<\rho$ implies $c<\frac{a}{b}$. Thus, a linear industry is described by the parameters $a, b, c$, a set of firms $N$ and a vector of capacities $k=\left(k_{1}, \ldots, k_{n}\right)$.

Delgado and Moreno [7] show that in a linear industry (without capacity constraints) $n \geq 3\left(=n^{*}\right)$ is a sufficient condition for the Cournot outcome to be the unique outcome that can be sustained by CPSFE.

\footnotetext{
${ }^{13}$ Note however that in Example 4.2 the Cournot outcome can also by sustained by CPSFE. Therefore, although the presence of asymmetries may allow for the existence of equilibrium outcomes other than Cournot, there is no guarantee that the resulting equilibrium price will be stictly lower than the Cournot price.
} 
Proposition 4.3 established that if additional CPSFE exist then, the number of firms which capacity is not binding at such equilibria must be less than $n^{*}$. Thus, in the case of a linear industry, any CPSFE leading to an outcome other than Cournot must involve at most two firms producing below their capacity. The search of additional CPSFE in a linear industry can then be restricted to the family of SFE in which less than three firms produce below their capacity.

Consider first the case where $\bar{n}=0$, i.e. no firm has enough capacity to produce the Cournot quantity. Then, from Corollary 2.2 and Theorem 3.3, there is a unique SFE outcome which is also the unique CPSFE outcome.

Next, consider the case where $0<\bar{n}<n^{*}=3$. The capacity-constrained Cournot outcome involves the $n-\bar{n}$ smallest firms producing at full capacity and the $\bar{n}$ largest firms producing the (unconstrained) Cournot output on the "residual demand" (see the definition of capacity-constrained Cournot outcome in section 2.2.) Consider the game faced by Firms $\{1, \ldots, \bar{n}\}$ when the $n-\bar{n}$ smallest firms produce at full capacity. Firms $\{1, \ldots, \bar{n}\}$ confront the residual linear industry $\left(D_{k, \bar{N}}, C, \bar{N}, k\right)$. In a linear industry $\left(D_{k, \bar{N}}, C, \bar{N}\right)$, where $\bar{n}<3$, there are several outcomes that can be sustained by CPSFE. In particular, any equilibrium outcome that is not Pareto-dominated by the Cournot outcome constitutes a CPSFE. As some of those equilibria may also be sustained by SFE in the industry $\left(D_{k, \bar{N}}, C, \bar{N}, k\right)$, the existence of a unique CPSFE is not guaranteed for $0<\bar{n}<n^{*}$.

Most interestingly, consider finally the case where $n^{*} \leq \bar{n}$. Let us start with the case of a linear industry where $\bar{n}=n^{*}=3$, i.e. there are three firms in the industry and the capacities of all three are above the Cournot quantity. Figure 1 illustrates the combination of capacities for which there is a unique CPSFE (which results in the Cournot outcome.) The horizontal axis represents the capacity of the largest firm, i.e. Firm 1, while the vertical axis represents the capacity of Firm 3. The shaded area represents the combination of capacities for which a unique CPSFE outcome exists (which includes the case of symmetric capacities, i.e. $k_{1}=k_{2}=k_{3}$.) Note that, since by assumption $k_{1} \geq k_{3}$, only the area below the 45-degree line is relevant.

In this area, either the capacity of the largest firm is not sufficiently large (in comparison to the capacity of the smallest firm) and all possible SFE result in profits lower than the Cournot profits (Area B) or the capacity of the smallest firm 


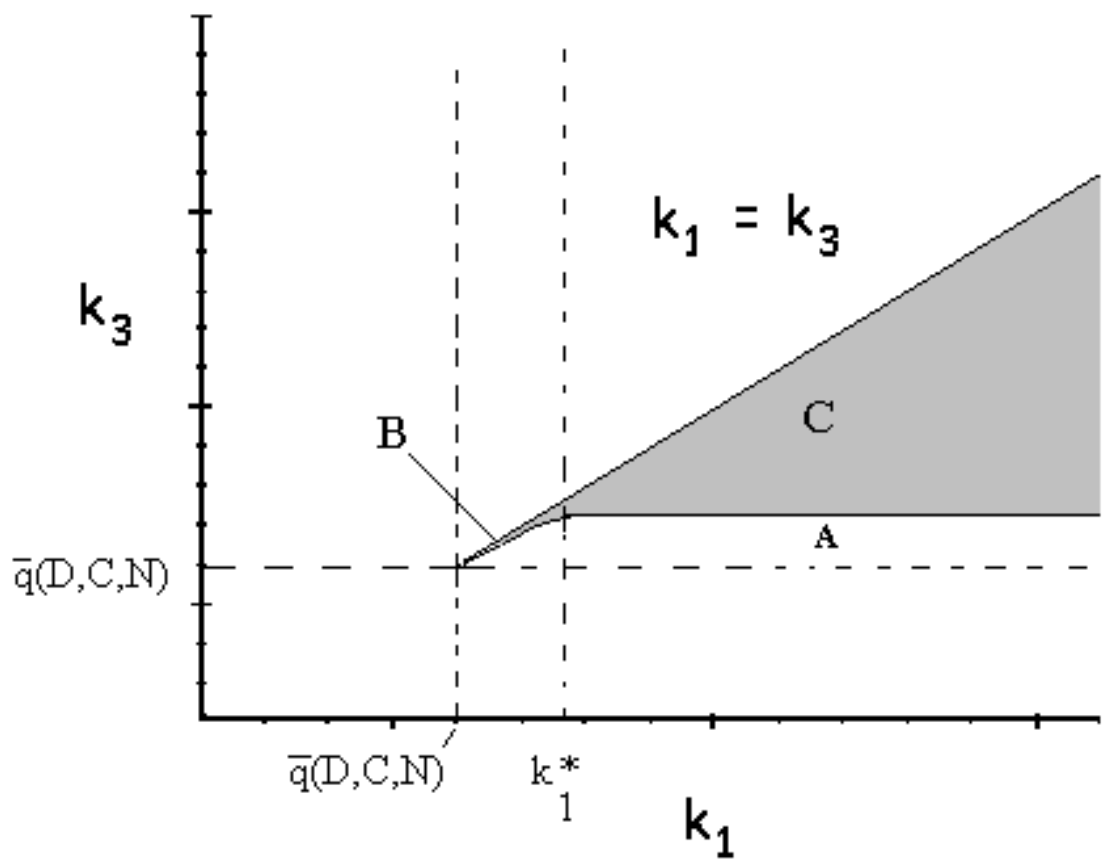

Figure 1:

is sufficiently large and there is no room to obtain above-Cournot profits when the capacity of Firm 3 is binding at equilibrium (Area C). In Areas B and C, the capacityconstrained Cournot outcome is the only market outcome that can be sustained by CPSFE. In the area below the shaded area (Area A), where the capacity of Firm 3 is sufficiently small, the uniqueness of a CPSFE outcome is not guaranteed and, in fact, outcomes other than the Cournot outcome can be sustained by CPSFE.

Theorem 4.4 formalizes the previous illustration for any $\bar{n} \geq n^{*}=3$. In particular, Theorem 4.4 provides necessary and sufficient conditions for the Cournot outcome to be the unique outcome that can be sustained by CPSFE under capacity constraints for $\bar{n} \geq n^{*}$. As shown in Figure 1, such conditions imply that firms are "sufficiently" symmetric.Denote $\widehat{k}=\frac{\sqrt{2}}{n+1}(a-b c)$. The following conditions linking the capacities of the different firms in the industry will be useful for establishing Theorem 4.4:

- Condition 4.4.1. $k_{1}<\widehat{k}$ and $\sum_{i>2} k_{i}>a-b c-k_{1}-\frac{\widehat{k}^{2}}{k_{1}}$;

- Condition 4.4.2. $k_{1} \geq \widehat{k}$ and $\sum_{i>2} k_{i}>a-b c-2 \widehat{k}$. 
Conditions 4.4.1 and 4.4.2 describe respectively regions $\mathrm{B}$ and $\mathrm{C}$ in Figure 1. Under 4.4.1 the largest firm is "too small" whereas under 4.4.2 the smaller firms are "too big". In both regions, any SFE is areto-dominated by the Cournot outcome. If capacities fall out of such regions CPSFE resulting in outcomes other than the Cournot outcome exist. The proof of this result is given in the Appendix.

Theorem 4.4. Let $(D, C, N, k)$ be a capacity-constrained linear industry where $\bar{n} \geq$ 3. The capacity-constrained Cournot outcome is the unique CPSFE outcome if and only if either 4.4 .1 or 4.4 .2 holds.

The following Remark shows that in linear industries Proposition 4.1 is a particular case of Theorem 4.4 since under symmetric capacity constraints either Condition 4.4.1 or Condition 4.4.2 holds. The proof is mechanical and is therefore omitted.

Remark 4.5. Consider the linear industry $(D, C, N, k)$ where $n \geq 3 ; k=\left(k_{1}, \ldots, k_{1}\right)$ and $N \equiv \bar{N}$. Then, either Condition 4.4.1 or Condition 4.4.2 holds and therefore the Cournot outcome is the unique outcome that can be sustained by CPSFE.

The following examples show some cases where either 4.4.1 or 4.4.2 does not hold and therefore there are CPSFE resulting in outcomes other than Cournot.

Example 4.6. Recall Example 4.2. Note that $\bar{q}=25<k_{1}=35<\widehat{k}=25 \sqrt{2}$ but $k_{3}=28<100-k_{1}-\frac{\widehat{k}^{2}}{k_{1}}=\frac{205}{7}$ (i.e. Condition 4.4.1 does not hold.) Example 4.2 shows that there is a strategy $\hat{s} \in \operatorname{CPSFE}(D, C,\{1,2,3\}, k)$ which leads to a price lower than the Cournot price.

Example 4.7. Consider the industry $(D, C,\{1,2,3\}, k)$ where $C(q)=0$ for $q \in \mathbb{R}$, $D(p)=100-p$ for $p \in[0,100]$, and $k=(40,40,28)$. Note that $k_{1}=40>\widehat{k}=25 \sqrt{2}$ but $k_{3}=28<100-2 \widehat{k}=29.28$ (i.e. Condition 4.4 .2 does not hold.)

As shown in the proof of Theorem 4.4, the strategy $\hat{s}$ such that

$$
\begin{aligned}
& \hat{s}_{1}(p)=36 \quad \forall p, \\
& \hat{s}_{2}(p)=\left\{\begin{array}{cc}
p & \text { if } p<30, \\
30 & \text { if } p \geq 30,
\end{array}\right. \\
& \hat{s}_{3}(p)=28 \quad \forall p .
\end{aligned}
$$

resulting in the outcome

$$
\left(p(\hat{s}), q_{1}(\hat{s}), q_{2}(\hat{s}), q_{3}(\hat{s})\right)=(18,36,18,28)
$$


constitutes a CPSFE.

Note that the proof of the sufficient conditions of Theorem 4.4 is based on the fact that the unique CPSFE outcome (i.e., the Cournot outcome) is Pareto-optimal within the set of SFE outcomes. This will happen for any demand/cost structure when capacities exceed some threshold. Therefore, for sufficiently large capacities (no matter how asymmetric they are), the Cournot outcome is the only outcome that can be sustained by CPSFE.

\section{Policy Implications}

The above results show that the standard industry concentration measures (such as the Herfindahl index,) whether applied to the distribution of the industry capacity or to the market shares, might not be useful to determine the level of prices in a market. As shown, in a market where firms compete via supply functions and are capacity constrained, some asymmetric distributions of capacities may give rise to equilibria where market shares are asymmetric and the market price is below the Cournot price, whereas symmetric distributions of capacities (which imply lower industry concentration) give rise to the Cournot price. Therefore, the number of firms being constant, more concentrated industry structures may give rise to lower market prices than less concentrated market structures.

The next subsections discuss the implication of this result in connection to two policy problems: the break-up of a monopoly and merger policy.

\subsection{Policy guidelines to break up a monopoly}

Consider a policymaker facing the problem of breaking up a monopoly into multiple firms in order to introduce competition. ${ }^{14}$ Assume the number of firms is determined exogenously and the policymaker has to decide on how to allocate the existing capacity among them. Assume the objective of the policymaker is to induce the lowest possible price. What can the policymaker learn from the previous results? ${ }^{15}$

\footnotetext{
${ }^{14}$ This was, for example, the problem faced by the UK government when privatizing and liberalizing the electricity generation industry that was previously a state monopoly.

${ }^{15}$ I thank an anonymous referee for suggesting the current format of this example.
} 
The policymaker has basically three alternatives: ${ }^{16}$ Either (a) allocating capacities in such a way that some firms cannot produce the 'unconstrained' Cournot quantity; or (b) allocating capacities asymmetrically in such a way that all firms can produce at least the Cournot output and there are multiple CPSFE outcomes; or (c) allocating capacities (quasi) symmetrically in such a way that all firms can produce at least the Cournot output and there is a unique CPSFE output. Note that the highest equilibrium price (i.e. the capacity-constrained Cournot price) will be higher in case (a) than in cases (b) or (c) -see Theorem 2.1. In addition, note that option (b) may yield other equilibrium prices which are below the Cournot price while in option (c) there would be a unique equilibrium price. Therefore, option (b) seems the right choice since in the worse case it will yield a price equal to option (c) but it could also yield lower prices.

Thus, the policymaker should on the one hand allow every firm to have sufficient capacity to produce the Cournot output and, on the other hand, allocate the total capacity asymmetrically in order to allow for the existence of multiple equilibria. This is easily shown by means of an example.

Example 5.1. Consider an industry where demand is given by $D=100-p$ for $p \in[0,100]$ and costs are null for any production level. Consider there is a monopolist with 93 units of capacity that has to be split-up into three firms. The capacity allocations $(36,36,21),(35,30,28)$ and $(31,31,31)$ are examples of options (a), (b) and (c) above respectively. The maximum equilibrium prices yielding from each alternative are $p=27$ for option (a) and $p=25$ for options (b) and (c). Prices below the Cournot price, such as $p=21$, can also yield in option (b) (see Example 4.2) while $p=25$ is the only equilibrium price in option (c). Therefore, the highest price yielding from option (b) is not higher than the ones yielding from options (a) and (c) and, since in option (c) there are multiple equilibria, the price could be lower. Thus, options (b) would seem the best choice.

\footnotetext{
${ }^{16}$ To simplify, it will be assumed that the total capacity is larger than the Cournot output.
} 


\section{$5.2 \quad$ Merger policy}

The results of this paper are also helpful for merger policy analysis. They indicate that, in some cases, mergers leading to asymmetric industry structures may yield prices lower than mergers leading to symmetric industry structures. This is illustrated by Example 5.2.

Example 5.2 also illustrates the possibility that the post-merger equilibrium price be lower than the pre-merger equilibrium price (even in the absence of efficiency gains.) The explanation of this result is that when the firms resulting from a merger are sufficiently asymmetric, prices below the Cournot price can be sustained by CPSFE, and that such prices may be even lower than the Cournot price of the premerger industry.

Example 5.2. Consider the industry $(D, C,\{1,2,3,4\}, k)$ where $C(q)=0$ for $q \in \mathbb{R}$, $D(p)=100-p$ for $p \in[0,100]$, and $k=(35,30,28,16)$. Note that firm 4 does not have enough capacity to produce the (unconstrained) Cournot quantity (which is equal to 20) and then $\bar{N}=\{1,2,3\}$. The industry $\left(D_{k,\{1,2,3\}}, C,\{1,2,3\}\right)$ where $D_{k,\{1,2,3\}}(p)=84-p$ satisfies the conditions in Theorem 4.4 and therefore the capacity constrained-Cournot outcome

$$
\left(\bar{p}, \bar{q}_{1}, \bar{q}_{2}, \bar{q}_{3}, \bar{q}_{4}\right)=(21,21,21,21,16)
$$

is the only outcome that can be sustained by CPSFE.

Assume firms 1 and 4 merge. Then the industry becomes $\left(D, C,\{1,2,3\}, k^{\prime}\right)$ where $k^{\prime}=(51,30,28)$. The outcome

$$
\left(p^{\prime}, q_{1}^{\prime}, q_{2}^{\prime}, q_{3}^{\prime}\right)=(18,36,18,28)
$$

is a possible CPSFE outcome -see Example 4.7. Note that $p^{\prime}<\bar{p}$, i.e. the equilibrium price after the merger is lower than the price before the merger. After the merger, there are multiple outcomes that can be sustained by CPSFE and some of them yield prices lower than the pre-merger price. Therefore, even in the absence of efficiency gains, a merger does not necessarily imply higher prices.

Turning back to the initial situation, consider now the merger of firms 3 and 4. Then the industry becomes $\left(D, C,\{1,2,3\}, k^{\prime \prime}\right)$ where $k^{\prime \prime}=(44,35,30)$. In this 
industry the capacity-constrained Cournot outcome

$$
\left(\bar{p}^{\prime \prime}, \bar{q}_{1}^{\prime \prime}, \bar{q}_{2}^{\prime \prime}, \bar{q}_{3}^{\prime \prime}\right)=(25,25,25,25)
$$

is the only outcome that can be sustained by CPSFE (since conditions 4.4 .1 and 4.4.2 are satisfied.) Note that this merger would result in a more symmetric industry structure than the previous one and would lead to a unique CPSFE outcome implying a higher price. This merger would also yield a price higher than the pre-merger price. ${ }^{17}$

The previous example shows that (a) mergers leading to asymmetric industry structures yield prices not higher than mergers leading to symmetric industry structures; and (b) if the post-merger capacities are sufficiently asymmetric, the postmerger prices may be even lower than the pre-merger prices (even in the absence of efficiency gains. $)^{18}$

This implies that one of the standard remedies of merger policy such as capacity divestiture through the transfer of capacity to smaller competitors may not always be appropriate since this measure increases symmetry and may lead to the existence of a unique outcome that can be sustained by CPSFE.

Capacity asymmetries have been shown to have similar effects in different contexts. Comte et al [6] analyze a model of Bertrand-Edgeworth price competition with exogenous capacity constraints and show that asymmetric distributions of capacities make collusive outcomes more difficult to sustain in an infinite period game. They conclude that mergers increasing capacity asymmetry may result in lower prices. Their conclusions are therefore in line with the previous example.

In practice policymakers do not always take into account the particularities of market design when analyzing the existence of market power and, in particular, in the analysis of mergers. For example, in Europe the analysis of mergers in the electricity industry (where firms compete via supply functions) has basically relied on standard concentration indices. ${ }^{19}$ In the US, the energy regulator FERC has detected the difficulty of predicting market outcomes from industry structure in electricity wholesale

\footnotetext{
${ }^{17}$ Note however that $\bar{p}^{\prime}$ (which equals $\bar{p}^{\prime \prime}$ ) may also be sustained by CPSFE in the industry $\left(D, C,\{1,2,3\}, k^{\prime}\right)$.

${ }^{18}$ Note however that, since multiple equilibria exist, this result depends on which market equilibrium yields.

${ }^{19}$ See, for example, cases IV/M.1606 EDF/South Western Electricity, IV/M.1803 Electrabel/EPON, COMP/M.3007 E.ON/TXU Europe Group and COMP/M.2209 EDF Group/Cottam
} 
markets and has proposed specific rules for the assessment of market power in such markets (see, e.g., section VI in [8].)

\section{Appendix}

Proof of Proposition 4.1. Let $s \in S F E(D, C, N, k)$ such that $p(s)<\bar{p}$. Without loss of generality, let $k=\left(k_{1}, \ldots, k_{1}\right)$.

Assume $k_{1} \leq \bar{q}(D, C, N)$. Then, there is a unique outcome that can be sustained by SFE, in which all firms produce at full capacity. That is, $p(s)=\bar{p}(D, C, N, k)$ and $q_{i}(s)=\bar{q}(D, C, N, k)=k_{1}$ for all $s \in S F E(D, C, N, k)$ where $\bar{p}(D, C, N, k)=\bar{p}$ is the solution to the equation $n k_{1}=D(\bar{p})$.

Assume $k_{1}>\bar{q}(D, C, N)$.

Recall $N(s)=\left\{i \in N \mid q_{i}(s)<k_{i}\right\}$. Consider two possibilities:

First, suppose $N(s) \equiv N$, i.e., $q_{i}(s)<k_{1}$, for all $i \in N$. Then, the capacity constraints play no role and $s \in S F E(D, C, N)$. Since $n \geq n^{*}$, thus, $\bar{\pi}(D, C, N, k)=$ $\bar{\pi}(D, C, N)>\pi_{i}(s)$ for all $i \in N$ and then $s \notin C P S F E(D, C, N, k)$.

Next, assume $N(s) \subset N$. It is shown that for any $s \in S F E(D, C, N, k)$ one can construct a strategy $\hat{s}$ such that $\hat{s} \in S F E(D, C, N)$ and such that it results in the same market outcome as $s$, i.e. $p(\hat{s})=p(s)$ and $q_{i}(\hat{s})=q_{i}(s) \forall i \in N$. Since for $n \geq n^{*}$, the Cournot outcome Pareto-dominates any other equilibrium outcome in the industry $(D, C, N)$, then $\hat{s} \notin C P S F E(D, C, N)$ and, consequently, $s \notin C P S F E(D, C, N, k)$.

Recall equilibrium condition $E_{i}$ and write $\alpha_{i}=r_{i}^{\prime}(p)$. Condition $E_{i}$ becomes

$$
q_{i}+\left(D^{\prime}(p)-\sum_{\substack{j \in N(s) \\ j \neq i}} \alpha_{j}\right)\left(p-C^{\prime}\left(q_{i}\right)\right)=0, \quad \text { for } i \in N(s)
$$

and

$$
k_{1}+\left(D^{\prime}(p)-\sum_{j \in N(s)} \alpha_{j}\right)\left(p-C^{\prime}\left(k_{1}\right)-\lambda\right)=0, \quad \text { for } i \in N \backslash N(s) .
$$

Power Station. In EDF Group/Cottam Power Station it is mentioned that the fact that the market price is determined through a pool mechanism could require a closer analysis. However, since no dominant position is said to be created or strengthened in any case, no further analysis is performed. 
I then construct a strategy $\hat{s} \in S F E(D, C, N)$ such that $p(\hat{s})=p(s)$ and $q_{i}(\hat{s})=$ $q_{i}(s) \forall i \in N$. For $i \in N$, consider the system of equations

$$
\hat{q}_{i}+\left(D^{\prime}(\hat{p})-\sum_{j \neq i} \hat{\alpha}_{j}\right)\left(\hat{p}-C^{\prime}\left(\hat{q}_{i}\right)\right)=0
$$

and set $\hat{p}=p(s), \hat{q}_{i}=q_{i}(s) \forall i \in N(s)$ and $\hat{q}_{i}=k_{1}$ for $i \in N \backslash N(s)$.

Thus,

$$
\begin{aligned}
\sum_{j \neq i} \hat{\alpha}_{j} & =\frac{p-C^{\prime}\left(k_{1}\right)-\lambda}{p-C^{\prime}\left(k_{1}\right)} \sum_{j \in N(s)} \alpha_{j}, \quad \text { for } i \in N \backslash N(s), \\
\sum_{j \neq i} \hat{\alpha}_{j} & =\sum_{\substack{j \in N(s) \\
j \neq i}} \alpha_{j} \quad \text { for } i \in N(s) .
\end{aligned}
$$

The system of equations 4.2 is a linear system with $n$ equations and $n$ unknowns. I show that the system has a unique solution $\left(\hat{\alpha}_{1}, \ldots, \hat{\alpha}_{n}\right)$ where $\hat{\alpha}_{i} \geq 0$ for $i \in N$, thereby showing that the system of equations 4.1 characterizes a SFE and, consequently, $\hat{s} \in \operatorname{SFE}(D, C, N)$.

Note that $\hat{\alpha}_{i}=\hat{\alpha}_{j}=\hat{\alpha} \forall i, j \in N \backslash N(s)$ and that, since $q_{i}(s)<k_{1} \forall i \in N(s)$, we have $\hat{\alpha}_{i}>\hat{\alpha}$ for $i \in N(s)$. Write $\phi=\frac{p-C^{\prime}\left(k_{1}\right)-\lambda}{p-C^{\prime}\left(k_{1}\right)}$. Note that $0<\phi \leq 1$. Rewrite system 4.2 as a system of $n(s)+1$ equations given by

$$
\begin{aligned}
\sum_{j \in N(s)} \hat{\alpha}_{j}+(n-n(s)-1) \hat{\alpha} & =\phi \sum_{j \in N(s)} \alpha_{j}, \\
\sum_{\substack{j \in N(s) \\
j \neq i}} \hat{\alpha}_{j}+(n-n(s)) \hat{\alpha} & =\sum_{\substack{j \in N(s) \\
j \neq i}} \alpha_{j} \quad \forall i \in N(s) .
\end{aligned}
$$

Hence, adding equations $4.2 b_{i}$ over $i \in N(s)$ yields the equation

$$
(n(s)-1) \sum_{j \in N(s)} \hat{\alpha}_{j}+n(s)(n-n(s)) \hat{\alpha}=(n(s)-1) \sum_{j \in N(s)} \alpha_{j} .
$$

Solving for $\sum_{j \in N(s)} \hat{\alpha}_{j}$,

$$
\sum_{j \in N(s)} \hat{\alpha}_{j}=\sum_{j \in N(s)} \alpha_{j}-(n-n(s))\left(1+\frac{1}{(n(s)-1)}\right) \hat{\alpha},
$$

and substituting in equation $4.2 a$,

$$
\hat{\alpha}=\frac{n(s)-1}{n-1}(1-\phi) \sum_{j \in N(s)} \alpha_{j},
$$


and since $0<\phi \leq 1$, then $\hat{\alpha} \geq 0$. Thus, since as $\hat{\alpha}_{i}>\hat{\alpha}$ for $i \in N(s)$, then $\hat{\alpha}_{i}>0 . \square$

Example 4.2. The strategy profile $\hat{s}$ is self-enforcing if $\hat{s} \in \operatorname{SFE}(D, C,\{1,2,3\}, k)$ and if for all $i \in N, \hat{s}_{N \backslash\{i\}} \in C P S F E\left(D_{\hat{s}, N \backslash\{i\}}, C, N \backslash\{i\}, k\right)$ where $D_{\hat{s}, N \backslash\{i\}}(p)=$ $D(p)-\hat{s}_{i}(p)$. Finally, $\hat{s}_{N \backslash\{i\}} \in C P S F E\left(D_{\hat{s}, N \backslash\{i\}}, C, N \backslash\{i\}, k\right)$ if $\hat{s}_{N \backslash\{i\}} \in S F E\left(D_{\hat{s}, N \backslash\{i\}}, C, N \backslash\{i\}, k\right)$ and there is no $\tilde{s} \in S F E\left(D_{\hat{s}, N \backslash\{i\}}, C, N \backslash\{i\}, k\right)$ satisfying $\pi_{i}(\tilde{s})>\pi_{i}(\hat{s})$ for $i \in N$.

It is easy to show that $\hat{s}$ is self-enforcing. It is already known that $\hat{s} \in S F E(D, C,\{1,2,3\}, k)$. It is mechanical to show that fixing Firm $i$ 's strategy, there exists no $\tilde{s} \in S F E\left(D_{\hat{s}, N \backslash\{i\}}, C, N \backslash\{i\}, k\right)$ satisfying $\pi_{i}(\tilde{s})>\pi_{i}(\hat{s})$ for $i \in N$.

Next, to show that there is no self-enforcing strategy $\tilde{s} \in S F E(D, C,\{1,2,3\}, k)$ such that $\pi_{i}(\tilde{s})>\pi_{i}(\hat{s})$ for $i \in N$, one has to show that there is no strategy $\tilde{s} \in$ $\operatorname{SFE}(D, C,\{1,2,3\}, k)$ such that $\pi_{i}(\tilde{s})>\pi_{i}(\hat{s})$ for $i \in N$.

Since $\hat{s}$ is self-enforcing and there is no self-enforcing strategy $\tilde{s}$ such that $\pi_{i}(\tilde{s})>$ $\pi_{i}(\hat{s})$ for $i \in N$, then $\hat{s} \in C P S F E(D, C,\{1,2,3\}, k)$.

Proof of Proposition 4.3. The proof follows immediately from the definition of CPSFE: if a strategy profile is a CPSFE, then such strategy profile should also be a CPSFE of the game played by any subcoalition of players.

Let $s \in S F E(D, C, N, k)$. Recall $N(s)=\left\{i \in N \mid q_{i}(s)<k_{i}\right\}$ and $n(s)=$ $\# N(s)$. Define

$$
D_{s, N(s)}(p)= \begin{cases}D(p)-\sum_{i \in N \backslash N(s)} k_{i} & \text { if } D(p)-\sum_{i \in N \backslash N(s)} k_{i} \geq 0, \\ 0 & \text { otherwise. }\end{cases}
$$

Let $s \in C P S F E(D, C, N, k)$ be such that $p(s)<\bar{p}\left(D_{k, \bar{N}}, C, \bar{N}\right)$ and $n(s) \geq n^{*}$. Consider the coalition $M \equiv N(s)$, write $m$ for the cardinality of $M$ and write $s_{M}$ for the strategy profile of the members of $M$. Holding fixed the strategies of the members of the complementary coalition, $s_{N \backslash M}$, the situation the group of firms in $M$ faces can be modeled as that of an industry $\left(D_{s, M}, C, M, k\right)$. It is shown that the members of the coalition $M$ can all improve by deviating to a strategy resulting in the Cournot outcome. Therefore, $s \notin C P S F E\left(D_{s, M}, C, M, k\right)$ and by definition $s \notin C P S F E(D, C, N, k)$.

Since $m=n(s) \geq n^{*}$, Delgado and Moreno [7] show that,

$$
p(s) q(s)-C(q(s))<\pi\left(\bar{p}\left(D_{s, M}, C, M\right)\right),
$$


and since $D_{s, M}(p)=D(p)-\sum_{i \in N \backslash M} k_{i} \geq D(p)-\sum_{i \in N \backslash M} k_{i}-\sum_{i \in M \backslash \bar{M}} k_{i}$, where $\bar{M}$ are the firms in $M$ that have enough capacity to produce the Cournot quantity, then, $\forall i \in \bar{M}$

$$
p(s) q_{i}(s)-C\left(q_{i}(s)\right)<\pi\left(\bar{p}\left(D_{s, M}, C, M\right)\right) \leq \pi_{i}\left(\bar{p}\left(D_{s, M}, C, M, k\right)\right) .
$$

Also, since, $\forall i \in M, q_{i}(s)<k_{i}$

$$
p(s) q_{i}(s)-C\left(q_{i}(s)\right)<\bar{p}\left(D_{s, M}, C, M, k\right) k_{i}-C\left(k_{i}\right), \quad \forall i \in M \backslash \bar{M} .
$$

Thus, $\pi_{i}\left(\bar{p}\left(D_{s, M}, C, M, k\right)\right)>p(s) q_{i}(s)-C\left(q_{i}(s)\right) \quad \forall i \in M$.

Then $s \notin C P S F E\left(D_{s, M}, C, M, k\right)$ and since $M \subset N$ then $s \notin C P S F E(D, C, N, k) . \square$

Proof of Theorem 4.4. First it is shown that if either condition 4.4.1 or condition 4.4.2 holds then the Cournot outcome Pareto-dominates any other SFE outcome and, since the Cournot outcome can be sustained by CPSFE, the Cournot outcome is the unique outcome that can be sustained by CPSFE.

Without loss of generality consider $\bar{n}=n .^{20}$ Note that the assumption $\bar{n}=n$, implies that every firm has enough capacity to produce the Cournot quantity of the industry without capacity constraints $\bar{q}(D, C, N)$ i.e., $k_{i} \geq \bar{q}(D, C, N)=\frac{a-b c}{n+1}$ for $i \in N$.

As shown in Proposition 4.3, any SFE at which there are three or more firms which capacity is not binding, is not CPSFE. Therefore, the search of additional CPSFE can be restricted to the family of SFE in which at least Firms $\{3, \ldots, n\}$ produce at full capacity. If it can be shown that no CPSFE leading to an outcome other than Cournot exists within this family, then identical result holds when any other $n-2$ firms produce at full capacity, since the "residual demand" left by any other combination of any $n-2$ firms will be smaller for each price.

Let $\tilde{s} \in S F E(D, C, N, k)$ where $q_{i}(\tilde{s})=k_{i}$ for $i>2,{ }^{21}$ and let $\hat{s} \in S F E\left(D_{k,\{1,2\}}, C,\{1,2\}, k\right)$, where $D_{\{1,2\}}(p)=D(p)-\sum_{i>2} k_{i}$, such that $\pi_{1}(\hat{s}) \geq$ $\pi_{1}(s)$ for all $s \in S F E\left(D_{k,\{1,2\}}, C,\{1,2\}, k\right)$. Note that, by definition of $\hat{s}, \pi_{1}(\hat{s}) \geq$ $\pi_{2}(\hat{s})$. It is shown that if any of the conditions of the theorem holds, then $\bar{\pi}(D, C, N)>$ $\pi_{1}(\hat{s}) \geq \pi_{i}(\tilde{s}) \forall i$. Thus, $\tilde{s} \notin C P S F E(D, C, N, k)$.

\footnotetext{
${ }^{20}$ If this assumption is not imposed, the proposition would hold by replacing the demand function $a-b p$ by the residual demand $a-b p-\sum_{i>\bar{n}} k_{i}$ and by using $\bar{n}$ instead of $n$.

${ }^{21}$ Note that if $a-b c-\sum_{i>2} k_{i} \leq 0$, then no SFE of this class exists.
} 
Assume Condition 4.4.1 holds. Then, $k_{1}<\frac{1}{2}\left(a-b c-\sum_{i>2} k_{i}\right)$. By Lemma A in the Appendix, $q_{1}(\hat{s})=k_{1} \geq k_{i} \forall i>2$. Then, $\pi_{1}(\hat{s}) \geq(p(\hat{s})-c) k_{i} \forall i>2$. By Lemma B in the Appendix, $\bar{\pi}(D, C, N)>\pi_{1}(\hat{s})$. Therefore, $\bar{\pi}(D, C, N)>\pi_{1}(\hat{s}) \geq$ $\pi_{i}(\tilde{s}) \forall i$. And thus, $\tilde{s} \notin C P S F E(D, C, N, k)$.

Assume Condition 4.4.2 holds. Then, $k_{1} \geq \frac{1}{2}\left(a-b c-\sum_{i>2} k_{i}\right)$. By Lemma A in the Appendix, $q_{1}(\hat{s})=\frac{1}{2}\left(a-b c-\sum_{i>2} k_{i}\right)$. Since $k_{1} \geq \frac{1}{2}\left(a-b c-\sum_{i>2} k_{i}\right)$ and $a-b c-\sum_{i>2} k_{i}>0$ then $k_{j}<\frac{1}{2}\left(a-b c-\sum_{i>2} k_{i}\right)=q_{1}(\hat{s})$ for $j \neq 1,2$. Then, $\pi_{1}(\hat{s}) \geq(p(\hat{s})-c) k_{i} \forall i>2$. By Lemma B in the Appendix, $\bar{\pi}(D, C, N)>\pi_{1}(\hat{s})$. Therefore, $\bar{\pi}(D, C, N)>\pi_{1}(\hat{s}) \geq \pi_{i}(\tilde{s}) \forall i$. And thus, $\tilde{s} \notin C P S F E(D, C, N, k)$.

Next, I show that if conditions 4.4 .1 and 4.4 .2 do not hold, then there exists at least one alternative outcome that can be sustained by CPSFE and does not yield the Cournot outcome, i.e., there exists $\hat{s} \in \operatorname{CPSFE}(D, C, N, k)$ such that $p(\hat{s})<\bar{p}(D, C, N, k)$. The proof is mechanical and will only be sketched.

Assume $k_{1}<\widehat{k}$ and $\sum_{i>2} k_{i} \leq a-b c-k_{1}-\frac{\widehat{k}^{2}}{k_{1}}$, i.e. condition 4.4.1 does not hold. Proceeding as in Example 4.2 -see Appendix- it can be proved that the strategy $\hat{s}$ such that

$$
\begin{aligned}
& \hat{s}_{1}(p)=k_{1} \quad \forall p, \\
& \hat{s}_{2}(p)= \begin{cases}b p & \text { if } p<\frac{k_{2}}{b} \\
k_{2} & \text { if } p \geq \frac{k_{2}}{b}\end{cases} \\
& \hat{s}_{i}(p)=k_{i} \quad \forall p \text { for } i>2
\end{aligned}
$$

is self-enforcing and that there is no self-enforcing strategy $\tilde{s}$ such that $\pi_{i}(\tilde{s})>\pi_{i}(\hat{s})$ for $i \in N$. Therefore $\hat{s} \in C P S F E(D, C, N, k)$.

Assume $k_{1} \geq \widehat{k}$ and $\sum_{i>2} k_{i} \leq a-b c-2 \widehat{k}$, i.e. condition 4.4 .2 does not hold. Proceeding as in Example 4.2 -see Appendix- it can be proved that the strategy $\hat{s}$ such that

$$
\begin{aligned}
& \hat{s}_{1}(p)=\frac{a-k_{3}-b c}{2} \quad \forall p \\
& \hat{s}_{2}(p)=\left\{\begin{array}{cc}
b p & \text { if } p<\frac{k_{2}}{b} \\
k_{2} & \text { if } p \geq \frac{k_{2}}{b}
\end{array}\right. \\
& \hat{s}_{i}(p)=k_{i} \quad \forall p \text { for } i>2
\end{aligned}
$$

is self-enforcing and that there is no self-enforcing strategy $\tilde{s}$ such that $\pi_{i}(\tilde{s})>\pi_{i}(\hat{s})$ for $i \in N$. Therefore $\hat{s} \in C P S F E(D, C, N, k)$. 
Lemma A. Let $(D, C,\{1,2\}, k)$ be a linear industry where $D(p)=a-b p-\Gamma$, $C(q)=c q, k=\left(k_{1}, k_{2}\right), \Gamma$ is an arbitrary constant such that $a-b c-\Gamma>0$ and $k_{i} \geq \bar{q}(D, C,\{1,2\})=\frac{a-b c-\Gamma}{3}$. Let $\left(p^{*}, q_{1}^{*}, q_{2}^{*}\right)$ be the outcome that maximizes Firm 1 's profits on the set $\operatorname{SFE}(D, C,\{1,2\}, k)$.

(a) If $k_{1} \geq \frac{1}{2}(a-b c-\Gamma)$, then $q_{1}^{*}=\frac{(a-b c-\Gamma)}{2}$ and $\pi_{1}\left(p^{*}, q_{1}^{*}, q_{2}^{*}\right)=\frac{1}{8 b}(a-b c-\Gamma)^{2}$.

(b) If $k_{1}<\frac{1}{2}(a-b c-\Gamma)$, then $q_{1}^{*}=k_{1}$ and $\pi_{1}\left(p^{*}, q_{1}^{*}, q_{2}^{*}\right)=\frac{1}{2}\left(\frac{a-b c-\Gamma-k_{1}}{b}\right) k_{1}$.

Proof. Delgado and Moreno [7] show that for every SFE of an industry $(D, C, N)$ one can find a SFE in a class in which all but (perhaps) one firm supply inelastically the output of the firm with the greatest profits (and output) at the original equilibrium. Moreover, this equilibrium leads to a price that is greater than or equal to the price associated to the original equilibrium. Consequently the profits of the firms using an inelastically supply are greater or equal than those of the firm with the largest profit at the original equilibrium.

This implies that when looking for Firm 1's profit maximizing equilibrium outcomes in the industry $(D, C, N)$ the search can be restricted to the SFE of this class. In the linear industry $(D, C,\{1,2\})$ these are characterized by the system of equations:

$$
\begin{aligned}
& q_{1}-(b+\alpha)(p-c)=0 \\
& q_{2}-b(p-c)=0 .
\end{aligned}
$$

Consider the linear industry $(D, C,\{1,2\}, k)$. Under capacity constraints, the system of equations becomes

$$
\begin{aligned}
q_{1}-(b+\alpha)(p-c) & \geq 0, \\
q_{2}-b(p-c) & \geq 0, \\
q_{1} & \leq k_{1}, \\
q_{2} & \leq k_{2} .
\end{aligned}
$$

where $A .1$ and $A .2$ hold with equality for $q_{1}<k_{1}$ and $q_{2}<k_{2}$ respectively. Therefore, using the market clearing condition, Firm 1's profit function can be written as a function of $q_{1}$ :

$$
\pi_{1}\left(q_{1}\right)=\left(\frac{a-b c-\Gamma-q_{1}}{2 b}\right) q_{1} \quad \text { for } \quad q_{1} \leq k_{1}
$$


Note that $\alpha \geq b\left(\frac{2 q_{1}}{1-q_{1}}-1\right)$ which is greater than or equal to zero for $q_{1} \geq \frac{a-b c}{3}$. Therefore, $\pi_{1}\left(q_{1}\right)$ is well defined for $q_{1} \geq \frac{a-b c}{3}$. Also note that $q_{2}=\frac{a-b c-q_{1}}{2}$ is decreasing in $q_{1}$ and $q_{2} \leq q_{1}$. Therefore, since $k_{2} \geq \frac{1}{3}$, Firm 2's capacity is never binding.

The function $\left(\frac{a-b c-\Gamma-q_{1}}{2 b}\right) q_{1}$ is increasing in $q_{1}$ and reaches its maximum at $q_{1}=$ $\frac{a-b c-\Gamma}{2}$. Therefore, if $k_{1} \geq \frac{a-b c-\Gamma}{2}, \pi_{1}\left(q_{1}\right)$ is maximum at $q_{1}=\frac{a-b c-\Gamma}{2}$ and $\pi_{1}\left(\frac{a-b c-\Gamma}{2}\right)=$ $\frac{1}{8 b}(a-b c-\Gamma)^{2}$. If $k_{1}<\frac{a-b c-\Gamma}{2}, \pi_{1}\left(q_{1}\right)$ is increasing for all feasible $q_{1}$ and therefore is maximized at $q_{1}=k_{1}$ and $\pi_{1}\left(k_{1}\right)=\left(\frac{a-b c-\Gamma-k_{1}}{2 b}\right) k_{1}$. $\square$

Lemma B. Let $(D, C, N, k)$ be a linear industry. Consider the industry $\left(D_{k,\{1,2\}}, C,\{1,2\}, k\right)$ where $D_{k,\{1,2\}}>0$. Let $\hat{s} \in \operatorname{SFE}\left(D_{k,\{1,2\}}, C,\{1,2\}, k\right)$ such that $\pi_{1}(\hat{s}) \geq \pi_{1}(s)$ for all $s \in S F E\left(D_{k,\{1,2\}}, C,\{1,2\}, k\right)$. If

B.1. $k_{1}<\widehat{k}$ and $\sum_{i>2} k_{i}>a-b c-k_{1}-\frac{\widehat{k}^{2}}{k_{1}}$, or

B.2. $k_{1} \geq \widehat{k}$ and $\sum_{i>2} k_{i}>a-b c-2 \widehat{k}$;

then $\pi_{1}(\hat{s})<\bar{\pi}(D, C, N)$.

Proof. Recall $\widehat{k}=\frac{\sqrt{2}}{n+1}(a-b c)$.

B.1. If $k_{1}<\widehat{k}$ and $\sum_{i>2} k_{i}>a-b c-k_{1}-\frac{\widehat{k}^{2}}{k_{1}}$, then $k_{1}<\frac{1}{2}\left(a-b c-\sum_{i>2} k_{i}\right)$. By Lemma A (replacing $\Gamma=\sum_{i>2} k_{i}$ ),

$$
\pi_{1}(\hat{s})=\frac{1}{2}\left(\frac{a-b c-\sum_{i>2} k_{i}-k_{1}}{b}\right) k_{1} .
$$

Using the conditions on $k_{1}$ and $\sum_{i>2} k_{i}$ :

$$
\begin{aligned}
\pi_{1}(\hat{s}) & <\frac{1}{2 b}\left(\left(a-b c-k_{1}\right)-\left(a-b c-k_{1}-\frac{\widehat{k}^{2}}{k_{1}}\right)\right) k_{1} \\
& =\frac{1}{b}\left(\frac{a-b c}{n+1}\right)^{2}=\bar{\pi}(D, C, N) .
\end{aligned}
$$

B.2. If $k_{1} \geq \widehat{k}$ and $\sum_{i>2} k_{i}>a-b c-2 \widehat{k}$, then $k_{1} \geq \frac{1}{2}\left(a-b c-\sum_{i>2} k_{i}\right)$. By Lemma A (replacing $\left.\Gamma=\sum_{i>2} k_{i}\right)$,

$$
\pi_{1}(\hat{s})=\frac{1}{8 b}\left(a-b c-\sum_{i>2} k_{i}\right)^{2} .
$$


Using the conditions on $k_{1}$ and $\sum_{i>2} k_{i}$,

$$
\begin{aligned}
\pi_{1}(\hat{s}) & <\frac{1}{8 b}\left(1-1+\frac{2 \sqrt{2}}{n+1}\right)^{2}(a-b c)^{2} \\
& =\frac{1}{b}\left(\frac{a-b c}{n+1}\right)^{2}=\bar{\pi}(D, C, N) .
\end{aligned}
$$

\section{References}

[1] Aumann, R.: Acceptable points in general cooperative $n$-person games. In: Contributions to the Theory of Games IV, Princeton, N.J.: Princeton Univ. Press (1959)

[2] Baldick, R., Hogan, W.: Capacity constrained supply function equilibrium models of electricity markets: stability, non-decreasing constraints, and function space iterations. University of California Energy Institute POWER working paper PWP-089 (2002)

[3] Bernheim, B., Peleg, B., Whinston, M.: Coalition-proof Nash equilibria: I concepts. Journal of Economic Theory 42, 1-12 (1987)

[4] Bernheim, B., Whinston, M.: Coalition-proof Nash equilibria: II applications. Journal of Economic Theory 42, 13-29 (1987)

[5] Chowdhury, P.R., Sengupta, K.: Coalition-proof Bertrand equilibria. Economic Theory 24, 307-324 (2004)

[6] Comte, O., Jenny, F., Rey, P.: Capacity constraints, mergers and collusion. European Economic Review 46, 1-29 (2002)

[7] Delgado, J., Moreno, D.: Coalition-proof supply function equilibria in oligopoly. Journal of Economic Theory 114, 231-254 (2004)

[8] Federal Energy Regulatory Commission: Order on rehearing and modifying interim generation market power analysis and mitigation policy, 107 FERC 
I 61,018. Available at http://www.ferc.gov/whats-new/comm-meet/041404/E1.pdf (2004)

[9] Ferreira, J. L.: A communication-proof equilibrium concept. Journal of Economic Theory 68, 249-257 (1996)

[10] Green, R., Newbery, D.: Competition in the British electricity spot market. Journal of Political Economy 100, 929-953 (1992)

[11] Grossman, S.: Nash equilibrium and the Industrial Organization of markets with large fixed costs. Econometrica 49, 1149-1172 (1981)

[12] Hart, O.: Imperfect competition in general equilibrium: an overview of recent work. In: Arrow, K., Honkaphoja, S. (eds.) Frontiers in Economics. Oxford: Basil Blackwell (1985)

[13] Klemperer, P., Meyer, M.: Supply function equilibria in oligopoly under uncertainty. Econometrica 57, 1243-1277 (1989)

[14] Konishi, H., Le Breton, M., Weber, S.: On coalition-proof Nash equilibria in common agency games. Journal of Economic Theory 85, 122-139 (1999)

[15] Kühn, K.-U.: Nonlinear pricing in vertically related duopolies. RAND Journal of Economics 28, 37-62 (1997)

[16] Noe, T.H.: Rationalizable and coalition-proof shareholder tendering strategies in corporate takeovers. Review of Quantitative Finance and Accounting 11, 269291 (1998)

[17] Wilson, R.: Auctions for shares. Quarterly Journal of Economics 93, 675-698 (1979) 